\title{
CYLINDRICALLY SYMMETRIC INHOMOGENEOUS MAXWELL'S FIELD WITH PEFECT FLUID IN BIMETRIC RELATIVITY
}

\author{
S. D. Deo ${ }^{1}$, Shalu D. Barai ${ }^{2}$, Nitu K. Gupta ${ }^{3}$ \\ N.S. Sc. And Arts College, Bhadrawati, Dist-Chandrapur, (M.S), \\ E-mail : $\underline{\text { s_deo01@yahoo.in }}$
}

\section{ABSTRACT :}

Cylindrically symmetric inhomogeneous space time is studied with the matter Maxwell's field with perfect fluid in the context of Rosen's bimetric theory of relativity and it is found that, in this theory and Maxwell's field as well as perfect fluid does not exist. And hence vacuum solutions can be obtained.

Keywords : Cylindrically, symmetric, inhomogeneous, Maxwell's field, perfect fluid bimetric relativity.

\section{INTRODUCTION:}

To remove some of the unsatisfactory features of the general theory of relativity, Rosen[1-3]proposed the biometric theory of relativity, in which there exits two metric tensors at each point of space time $g_{\mathrm{ij}}$ which describes gravitation, and the background metric $g_{\mathrm{ij}}$ which enters into the field equations and interacts with $g_{\text {ij }}$ but does not interact directly with matter.

Accordingly, at each space time point, one has two line elements

$$
\mathrm{ds}^{2}=g_{\mathrm{ij}} \mathrm{dx} \mathrm{x}^{\mathrm{i}} \mathrm{x} \mathrm{x}^{\mathrm{j}}
$$

And

$$
d \sigma^{2}=\gamma_{i j} d x^{i} d x^{j}
$$

where ds is the interval between two neighboring events as measured by a clock and a measuring rod. The interval $d \sigma$ is an abstract or geometrical quantity not directly measurable. One can regard it as describing the geometry that exists if no matter were present. 
Letelier [4] has studied spherical, plane and a particular case of cylindrical symmetry and given the general solution to Einstein's field eqations for cosmic cloud stings .

In 1991, Nevin [5] has solved Einstein's field eqations for spheri- cally or static cylindrically symmetric with string dust source. H.Baysalet al [6] has studied strings cosmological models in cylindrically symmetric inhomogeneous universe in general relativity and various physical and geometrical properties of the model have been discussed.

Mahurpawar and Deo [7] studied the cosmic strings in cylindrically symmetric cosmological model in bimetric relativity. And observed that the model does not exist in his theory.

Further Deo [8] has studied cylindrically symmetric inhomogeneous cosmic strings and domain walls in bimetric relativity.

Here, cylindrically symmetric inhomogeneous universe is studied with Maxwell's field coupled with perfect fluid in bimetric theory of relativity. and it is observe that in this theory Maxwell's field as well as perfect fluid does not exist. Hence one can obtain vacuum solutions.

\section{Field equations of bimetric relativity}

Field equations of bimetric relativity formulated by Rosen N [1-3] are

$K_{i}^{j}=N_{i}^{j}-\frac{1}{2} N g_{i}^{j}=-8 \pi k T_{i}^{j}$

Where

$$
N_{i}^{j}=\frac{1}{2} \square \mathrm{a} \beta\left[g^{\text {hj }} g_{\text {hi }}\right.
$$




$$
N=N_{\alpha}^{\alpha}, K=(g / \square)^{1 / 2} \quad g=\operatorname{det} .\left(g_{\mathrm{ij}}\right), \square=\operatorname{det} .(\square \text { ij })
$$

And a vertical bar (I) denotes the covariant differentiation with respect to $g_{\mathrm{ij}}$ $T_{i}^{j}$ is the energy momentum tensor for matter field.

\section{Cylindrically symmetric inhomogeneous perfect fluid and Maxwell's field solutions:}

Let us consider that cylindrically symmetric inhomogeneous space time in the form

$$
d s^{2}=A^{2}\left(d x^{2}-d t^{2}\right)+B^{2} d y^{2}+C^{2} d z^{2}
$$

where $A, B \& C$ are the functions of $\mathrm{x}$ and $\mathrm{t}$ and the background metric corresponding to the equation (3.1) is

$$
d \sigma^{2}=-d t^{2}+d x^{2}+d y^{2}+d z^{2}
$$

The energy momentum tensor $T_{i}^{j}$ for the matter Maxwell's field with perfect fluid is given by

$$
T_{i}^{j}=T_{i}^{j} \text { perfect fluid }+E_{i}^{j}
$$

where

$$
\mathrm{T}_{i \text { perfect fluid }}^{j}=(p+\epsilon) v_{i} v^{j}+p g_{i}^{j}
$$

Here $\epsilon$ is the energy density of the matter, $p$ is the pressure and $v_{i}$ is the flow vector together with the orthogonality condition

And

$$
v_{4} v^{4}=-1
$$

$$
\mathrm{E}_{\mathrm{i}} \mathrm{j}=\mathrm{F}_{\mathrm{ir}} \mathrm{F}^{\mathrm{jr}}-\frac{1}{4} \mathrm{~F}_{\mathrm{ab}} \mathrm{F}^{\mathrm{ab}} \mathrm{g}_{\mathrm{i}}
$$


Where $E_{j} j$ is the electromagnetic energy tensor and $F_{\text {ir }}$ is the Electromagnetic field tensos.

It is assume that the commoving system contains a magnetic field along the axis of symmetry. Only the components $F_{12}=-F_{21}$ of

The electromagnetic field tensor $F_{\text {ir }}$ are different from zero. And Maxwell's field equation

$$
F_{i j, k}+F_{j k, i}+F_{k i, j}=0
$$

give rise to

$$
\left.\mathrm{F}_{12}=-\mathrm{F}_{21}=\mathrm{F} \text { (a constant }\right)
$$

Using the equations (2.1)-(2.3) with (3.1)-(3.7) we get

$$
\begin{aligned}
& {\left[\left(\frac{B^{\prime \prime}}{B}-\frac{B^{2}}{B^{2}}\right)-\left(\frac{\ddot{B}}{B}-\frac{\dot{B}^{2}}{B^{2}}\right)+\left(\frac{C^{\prime \prime}}{C}-\frac{C^{2}}{C^{2}}\right)-\left(\frac{\ddot{C}}{C}-\frac{\dot{C}^{2}}{C^{2}}\right)\right]=-8 \pi k(W+2 \epsilon)} \\
& {\left[\left(\frac{B^{\prime \prime}}{B}-\frac{B^{2}}{B^{2}}\right)-\left(\frac{\ddot{B}}{B}-\frac{\dot{B}^{2}}{B^{2}}\right)-\left(\frac{C^{\prime \prime}}{C}-\frac{C^{2}}{C^{2}}\right)+\left(\frac{\ddot{C}}{C}-\frac{\dot{C}^{2}}{C^{2}}\right)-\left(\frac{A^{\prime \prime}}{A}-\frac{A^{2}}{A^{2}}\right)+\left(\frac{\ddot{A}}{A}-\frac{\dot{A}^{2}}{A^{2}}\right)\right]} \\
& =-8 \pi k(W+2 p) \\
& {\left[\left(\frac{B^{\prime \prime}}{B}-\frac{B^{2}}{B^{2}}\right)-\left(\frac{\ddot{B}}{B}-\frac{\dot{B}^{2}}{B^{2}}\right)+\left(\frac{C^{\prime \prime}}{C}-\frac{C^{2}}{C^{2}}\right)+\left(\frac{\ddot{C}}{C}-\frac{\dot{C}^{2}}{C^{2}}\right)-\left(\frac{A^{\prime \prime}}{A}-\frac{A^{2}}{A^{2}}\right)+\left(\frac{\ddot{A}}{A}-\frac{\dot{A}^{2}}{A^{2}}\right)\right]} \\
& =-8 \pi k(W+2 p) \\
& {\left[\left(\frac{B^{\prime \prime}}{B}-\frac{B^{2}}{B^{2}}\right)-\left(\frac{\ddot{B}}{B}-\frac{\dot{B}^{2}}{B^{2}}\right)+\left(\frac{C^{\prime \prime}}{C}-\frac{C^{2}}{C^{2}}\right)-\left(\frac{\ddot{C}}{C}-\frac{\dot{C}^{2}}{C^{2}}\right)\right]=-8 \pi k(W+2 \epsilon)} \\
& \text { where } \quad \dot{A}=\frac{\partial A}{\partial t}, \quad A=\frac{\partial^{2} A}{\partial t^{2}}, \quad A^{\prime}=\frac{\partial A}{\partial X} \quad \mathrm{~A}^{\prime \prime}=\frac{\partial^{2} \mathrm{~A}}{\partial \mathrm{X}^{2}} \quad \text { etc }
\end{aligned}
$$

In cylindrically symmetric inhomogeneous universe, the rotations $\omega^{2}$ is identically zero, the expansion $\theta$ sheer scalar $\sigma^{2}$ acceleration vector $\dot{v}_{i}$ and proper volume $\mathrm{V}^{3}$ are found respectively to have the following expressions: 
$\theta=V_{; i}^{j}=A^{-1}\left(\frac{\dot{A}}{A}+\frac{\dot{B}}{B}+\frac{\dot{C}}{C}\right)$

$\boldsymbol{\sigma}^{2}=\frac{1}{2} \sigma_{\mathrm{ij}} \mathrm{\sigma}^{\mathrm{ij}}=\frac{1}{3} \theta^{2}-\mathrm{A}^{-2}\left(\frac{\dot{\mathrm{A}} \dot{\mathrm{B}}}{\mathrm{AB}}+\frac{\dot{\mathrm{A}} \dot{\mathrm{C}}}{\mathrm{AC}}+\frac{\dot{\mathrm{B}} \dot{\mathrm{C}}}{\mathrm{BC}}\right)$

where

$\boldsymbol{\sigma}_{\mathrm{ij}}=\frac{1}{2}\left(V_{\mathrm{I}} ; \mathrm{V}_{\mathrm{j}}=+\mathrm{V}_{\mathrm{j}} ; \mathrm{i}\right)-\frac{1}{3} \theta^{2}\left(\mathrm{~g}_{\mathrm{ij}}-\mathrm{V}_{\mathrm{i}} \mathrm{V}_{\mathrm{j}}\right)$

And $\mathrm{V}_{\mathrm{i}}=\mathrm{V}_{\mathrm{i} ;} ; \mathrm{j} \mathrm{j}=\left(\frac{A^{\prime}}{A}, 0,0,0\right)$

Also $\mathrm{V}^{3}=\sqrt{(-g)}=A^{2} \mathrm{BC}$

Now using the equation (3.5),(3.6),(3.7)\&(3.10) we get

$\omega^{2} 16 \pi(\omega+p+\epsilon)=0$

But in view of reality conditions $\quad p>0, \quad \epsilon>0, W>0$ we have

$p=0=\epsilon=\mathrm{W}$

i.e. $\quad p=0=\epsilon$ and $\mathrm{w}=0$

Implies that $\frac{F^{2}}{A^{2} B^{2}}=0$

i.e. $F_{12}=-F_{21}=0$

Thus, one can state that the nil contribution of perfect fluid and Maxwell's field solution in cylindrically symmetric inhomogeneous universe in bimetric relativity.

\section{CONCLUSION:}

Here we have observed that Maxwell's field with perfect fluid solution does not exist in cylindrically symmetric inhomogeneous cosmological model in bimetric relativity further one can obtain vacuum solutions. 


\section{REFERENCES:-}

Rosen N. 1940, Phys. Rev. 57147

Rosen N. 1973, Gen. Rela.Grav. 6435

Rosen N. 1975, Gen. Rela.Grav. 6259

Letelier P.S. 1979, phys. Rev.D201294

Nevin J.N. 1991, Gen.Rela.Grav.23253

Baysal H. , Yavuz I. , Tarhan I. , Camci U. , Yilmaz I., Turk. J. , 2001, Phys. 25, 283

Mahurpawar V. , Deo S.D. 2002, The Nepali Math.Sc.Report 20, 43

Deo S.D. 2011, International J. of Mathematical Archive 2, 121 